\title{
Criminologie
}

\section{L’impact de la réaction policière sur l'évolution de vols avec violence}

\section{Daniel Élie et Anne Kapétanaki-Barake}

Volume 18, numéro 2, 1985

Le vol à main armée à Montréal

URI : https://id.erudit.org/iderudit/017216ar

DOI : https://doi.org/10.7202/017216ar

Aller au sommaire du numéro

\section{Éditeur(s)}

Les Presses de l'Université de Montréal

ISSN

0316-0041 (imprimé)

1492-1367 (numérique)

Découvrir la revue

Citer cet article

Élie, D. \& Kapétanaki-Barake, A. (1985). L'impact de la réaction policière sur l'évolution de vols avec violence. Criminologie, 18(2), 47-61.

https://doi.org/10.7202/017216ar

\section{Résumé de l'article}

This descriptive and comparative study analyses the effectiveness of police activity on the evolution of robbery and armed robbery in four Canadian provinces and five metropolitan areas. The statistical data used were those furnished by Statistics Canada on cases of robbery, armed robbery, the clearance rate and the number of persons charged with these crimes. The evaluation of police effectiveness was made by analysing the variation in the rate of solution on the rate of crime and the rate of the number of persons accused on the rate of crime.

The analysis showed that the influence of police action on the control of robbery and armed robbery, as expresses by the clearance rates is almost the same from one province or metropolitan area to another. Furthermore, the effectiveness of police action seems to depend on the volume of crime that the police have to cope with. Also, the analysis of the variation in the clearance rate and the rate of persons accused in relation to the rate of crime showed that an increase in the volume of crime is independent of the efforts made by the police. 


\section{L'IMPACT DE LA RÉACTION POLICIÈRE SUR L'ÉVOLUTION DES VOLS AVEC VIOLENCE Daniel Élie* Anne Kapétanaki-Barake**}

This descriptive and comparative study analyses the effectiveness of police activity on the evolution of robbery and armed robbery in four Canadian provinces and five metropolitan areas. The statistical data used were those furnished by Statistics Canada on cases of robbery, armed robbery, the clearance rate and the number of persons charged with these crimes. The evaluation of police effectiveness was made by analysing the variation in the rate of solution on the rate of crime and the rate of the number of persons accused on the rate of crime.

The analysis showed that the influence of police action on the control of robbery and armed robbery, as expresses by the clearance rates is almost the same from one province or metropolitan area to another. Furthermore, the effectiveness of police action seems to depend on the volume of crime that the police have to cope with. Also, the analysis of the variation in the clearance rate and the rate of persons accused in relation to the rate of crime showed that an increase in the volume of crime is independent of the efforts made by the police.

\section{INTRODUCTION}

Les études entreprises en vue de déterminer l'impact de l'action policière sur la criminalité de violence ont été inspirées par les deux types de modèles suivants : les modèles de simultanéité et les modèles de dissuasion.

1. Les modèles de simultanéité défendus par les économistes cherchent à identifier les déterminants de la criminalité. D'après Blumstein et al., (1978), les défenseurs du modèle de simultanéité considèrent que les ressources de la police jouent un rôle important si on veut déterminer l'efficacité de l'action policière. Ils postulent, par ailleurs, que le risque qu'un malfaiteur soit

- Professeur agrégé, École de criminologie, Université de Montréal.

** Criminologue, Centre des services sociaux du Montréal Métro. politain. 
appréhendé est positivement relié aux ressources de la police. Ainsi, une augmentation au niveau des ressources devrait aussi augmenter le risque encouru par les criminels d'être appréhendés, parce que alors la police aura la possibilité de consacrer plus d'efforts à la solution d'actes criminels. De plus, en tenant les ressources policières constantes, une augmentation de la criminalité devrait diminuer la capacité de la police à solutionner les crimes commis et par conséquent, le risque d'appréhension sera diminué. Le modèle assure en plus que les ressources assignées au système de la justice criminelle en général et à la police en particulier, devraient être positivement affectées par le taux de criminalité.

Avio et Clark (1974), à partir de données statistiques de différentes provinces canadiennes pour la période de 1970-1972, ont construit un modèle dans lequel, le taux de criminalité, le taux de solution et les dépenses de la police per capita sont reliés simultanément. Le modèle a été utilisé pour les vols qualifiés, les vols par effraction et les fraudes. Pour chaque catégorie de crimes, ils ont trouvé une relation négative et généralement significative entre le taux de solution et le taux de criminalité.

Philipps et Votey (1972) ont utilisé les données statistiques des crimes-indices pour la période 1953-1968. Ils ont également établi un modèle selon lequel le taux de solution et le taux de criminalité sont déterminés simultanément : eux aussi ont trouvé une relation négative significative entre le taux de solution et le taux de criminalité. Wellford (1974) s'employa à vérifier la relation entre les changements produits aux divers niveaux de criminalité et les changements de diverses variables socio-démographiques et celles du contrôle social. De plus, il analysa l'impact de l'effort de la police, mesuré par le taux de solution, sur le taux de criminalité pour la période de 1960-1971. Il a trouvé une relation faible entre le taux de criminalité et les efforts de la police.

2. Les modèles de dissuasion tentent d'évaluer les effets de sanctions imposées par les diverses instances de la Justice criminelle ainsi que de leurs influences sur la criminalité. Selon Blumstein et al. (1978), les théories de dissuasion sont basées sur la promesse que les sanctions sont des mobiles négatifs et que leur imposition sur les criminels appréhendés servent à en décourager d'autres à s'engager dans la carrière criminelle. Ainsi, toutes les 
théories de dissuasion prévoient une relation négative entre le taux de criminalité et les différents niveaux de sanctions. Les niveaux de sanctions sont mesurés, soit par la sévérité des sanctions, soit par le risque encouru par la personne qui s'engage dans la carrière criminelle ou les deux ensemble. Tittle (1969) a suggéré que la probabilité de la punition doit atteindre un niveau critique avant qu'un effet de dissuasion soit possible. Tittle et Rowe (1974) ont tenté d'identifier le point critique au niveau duquel la certitude de la punition est associée à une diminution du taux de criminalité. Dans ce but, ils ont analysé la relation entre la probabilité d'arrestation et le taux de criminalité dans les villes et comtés de Floride pour l'année 1971. Les données ont présenté une relation non linéaire avec un point critique où on atteint un niveau de $30 \%$ du taux de solution. Les auteurs postulent que ce point critique $(30 \%)$ doit être atteint avant qu'il y ait un changement notoire au volume de criminalité.

Barley (1976) a entrepris le même genre d'analyse pour l'année 1972. Il a examiné la relation entre le taux de criminalité et le taux d'arrestation pour la criminalité en général dans les villes et comtés de Floride. De plus, il a fait introduire une mesure de la force policière (nombre des policiers/1000 h) comme une variable contrôle en vue d'analyser la relation entre le taux de certitude d'être appréhendé et le taux des délits commis.

Les résultats obtenus par Bailey concordent avec ceux de Tittle et Rowe (1974). Cependant, les données ont indiqué que la menace d'arrestation n'a pas un effet de dissuasion uniforme pour tous les délits. Car pour les homicides, les viols et les vols qualifiés, le niveau des arrestations paraissent avoir peu d'influence.

Selon Greenbert et al. (1979), l'impact de la criminalité sur les actions de la police peut apparaître d'au moins deux façons :

1. Un taux de criminalité élevé peut contribuer à saturer les capacités de la police dans le contrôle à court terme de la criminalité.

2. Un taux de criminalité élevé peut créer une certaine accoutumance envers la criminalité et dès lors contribuer à des punitions moins sévères ou au contraire, augmenter la peur du public vis-à-vis de la criminalité obligeant ainsi les autorités à 
infliger des punitions plus sévères ou des mesures de contrôle plus efficaces.

Dans le but de vérifier ces postulats, les auteurs ont utilisé le Multiware panel models, lequel impose des hypothèses sur l'uniformité des effets plutôt que d'assurer que certains effets ont des valeurs données. L'analyse fut basée sur le taux des crimes rapportés et le taux de solution d'après les arrestations dans 98 villes américaines durant la période 1964-1970; ils ont trouvé que le taux d'arrestation n'a pas d'effets mesurables sur le taux de criminalité rapporté. Chilton (1982) pour tester les effets dissuasifs des arrestations, a comparé les tendances du taux de criminalité du taux d'arrestation et le rapport des arrestations/ criminalité et cela, pour 49 villes américaines au cours de la période de 1960 à 1977. Les résultats obtenus par Chilton infirment l'hypothèse de dissuasion. Cependant, ils indiquent que le rapport arrestation/criminalité fluctue selon les années et qu'il est affecté par le fait que les citoyens déclareront ou non un crime.

Dans l'ensemble, les études effectuées à ce jour ont cherché à déterminer soit l'impact de pratiques de la police sur la criminalité de violence en général, soit les effets de la criminalité sur les pratiques de la justice criminelle.

\section{DESCRIPTION DE L'ÉTUDE}

La présente étude a été effectuée dans le but de décrire et de comparer l'efficacité du contrôle policier vis-à-vis du vol qualifié et du vol à main armée. $\grave{A}$ cette fin, nous avons sélectionné les données statistiques disponibles pour dix-neuf années. Nous avons étudié la période de 1962 à 1980 pour les vols qualifiés et de 1975 à 1980 pour les vols à main armée.

Quatre provinces canadiennes (Québec, Ontario, Alberta et Colombie britannique) et cinq de leurs zones métropolitaines (Montréal, Toronto, Vancouver, Calgary et Edmonton) faisaient l'objet de notre étude. Les tendances des variables suivantes furent analysées.

1. Le nombre réel des V.Q. ${ }^{1}$ et des V.A.M.A. ${ }^{2}$

1. V.Q. : vol qualifié.

2. V.A.M.A. : vol à main armée. 
2. Le nombre réel des V.Q. et des V.A.M.A. solutionnés.

3. Les personnes accusées pour V.Q. et V.A.M.A.

4. L'évolution de la population dans les provinces et les zones métropolitaines choisies.

Pour définir ces variables, nous empruntons les définitions proposées par Statistique Canada. Ainsi, le nombre réel des V.Q. et des V.A.M.A. correspond aux délits communiqués à la police ou connus d'elle et dont on a soustrait ceux qui se sont avérés non fondés.

Par V.Q. et V.A.M.A. solutionnés, nous entendons ceux qui sont :

a) classés par mise en accusation : un Q.V. est classé ainsi lorsqu'au moins une personne a été dénoncée par la police;

b) classés sans mise en accusation : un Q.V. est classé comme tel lorsqu'un inclupé est envoyé dans une institution psychiatrique et qu'il est probable qu'il en sorte; lorsqu'une personne avoue avoir commis un délit et meurt avant d'être inculpée; etc.

Par personne accusée, nous entendons le nombre réel de personnes inculpées par la police pour avoir commis des V.Q. et des V.A.M.A. Dans le cas où une même personne a commis plusieurs délits, le décompte des accusations correspond au nombre de crimes. En ce qui concerne les effectifs de la police, nous avons considéré uniquement le nombre de policiers en uniforme.

Les données statistiques recueillies proviennent des rapports annuels de Statistique Canada et les rapports annuels de la police de la C.U.M. En fait, on a eu recours à trois catégories de sources : les statistiques de la criminalité, les statistiques de l'administration policière, les statistiques démographiques.

Pour normaliser nos données et éviter les disparités géographiques, nous avons exprimé le nombre réel V.Q. et le nombre réel V.A.M.A. en taux $/ 100000 \mathrm{~h}$. De même pour le nombre de personnes accusées. Les effectifs policiers ont été considérés d'après le taux d'encadrement policier/1 000 habitants.

Nous avons commencé, tout d'abord, par la description et la comparaison de l'évolution du taux de criminalité (V.Q., V.A.M.A.), le taux de solution et les personnes accusées par la 
police pour avoir commis ce genre de crimes dans les quatre provinces et les cinq zones métropolitaines. Ensuite nous avons procédé à l'évaluation de l'action policière. Nous avons mis en rapport les indicateurs de l'action policière (taux de solution et taux de personnes accusées) avec le taux de criminalité (V.Q. V.A.M.A.).

Une idée approximative de l'influence de l'action policière sur la criminalité devait nous être fournie par l'étude de la variation des rapports suivants :

le taux de solution / le taux des vols qualifiés

le taux de solution / le taux des vols à main armée et

le taux des personnes accusées / le taux des vols qualifiés

le taux des personnes accusées / le taux des vols à main armée

Dans le but de déceler l'influence du taux d'encadrement policier sur l'appréhension des personnes soupçonnées d'avoir commis des vols qualifiés ou des vols à main armée, nous avons exprimé le rapport Taux des personnes accusées sur Taux de criminalité par le taux d'encadrement policier. Or, nous n'avons pas constaté de changements notables dans la variation de ce rapport, ce qui laisse supposer que l'évolution de la criminalité influence peu les variations survenues dans le taux d'encadrement policier durant la période étudiée. Cependant, nous devons mentionner qu'aucune hypothèse n'avait été préalablement formulée à propos du rôle du nombre des policiers sur la criminalité.

\section{OBSERVATION ET DISCUSSION}

En ce qui concerne l'évolution de la criminalité et de l'action policière durant ces dix-neuf années, les données ont révélé que les provinces et zones métropolitaines où les vols qualifiés et les vols à main armée sont moins nombreux, les corps policiers enregistrent un taux relativement élevé de vols qualifiés et de vols à main armée solutionnés.

De plus, il s'est avéré que les taux moyens de vols à main armée solutionnés par les corps policiers dans les quatre provinces et les cinq zones métropolitaines, sont légèrement supérieurs à ceux des vols qualifiés résolus (voir tableaux 1 et 2 ). 
TABLEAU 1

Taux de solution moyen des vols qualifiés et des vols d main armée selon les provinces

\begin{tabular}{lcccc}
\hline & Québec & Ontario & Alberta & $\begin{array}{c}\text { Colombie } \\
\text { britannique }\end{array}$ \\
\hline Vols qualifiés & $26,2 \%$ & $41,3 \%$ & $41,7 \%$ & $30,6 \%$ \\
\hline Vols à main armé & $25,4 \%$ & $44,8 \%$ & $43,8 \%$ & $37,3 \%$ \\
\hline
\end{tabular}

TABLEAU 2

Taux de solution moyen des vols qualifiés des vols à main armée selon les zones métropolitaines

\begin{tabular}{lccccc}
\hline & Montréal & Toronto & Vancouver & Calgary & Edmonton \\
\hline $\begin{array}{l}\text { Vols qualifiés } \\
22,3 \%\end{array}$ & $41,6 \%$ & $27,5 \%$ & $42,3 \%$ & $40,0 \%$ \\
\hline $\begin{array}{l}\text { Vols à main } \\
\text { armée }\end{array}$ & $22,0 \%$ & $43,7 \%$ & $33,1 \%$ & $45,7 \%$ & $39,7 \%$ \\
\hline
\end{tabular}

Les corps policiers du Québec et de la Communauté urbaine de Montréal enregistrent les taux de solution moyens les plus faibles comparativement à ceux des corps policiers des autres provinces et zones métropolitaines. Parallèlement, ils font face à un taux très élevé de vols à main armée et de vols qualifiés.

Par ailleurs, les corps policiers de l'Ontario et de la zone métropolitaine de Toronto présentent des taux moyens de crimes solutionnés plus élevés que ceux des autres, avec des taux de criminalité relativement faibles.

En Alberta et dans ses deux zones métropolitaines considérées, les corps de police accusent à la fois des taux de criminalité et des taux de solution élevés. Finalement, en Colombie britannique et dans la zone de Vancouver, la police enregistre un taux de criminalité et un taux de crimes solutionnés relativement élevés.

Il ressort, d'une part, que l'impact de l'action policière sur le contrôle des vols qualifiés et des vols à main armée, en matière du taux de solution, joue de façon presque semblable d'une province ou d'une zone métropolitaine à l'autre et, d'autre part, que l'efficacité de l'action entreprise semble dépendre grandement du volume de criminalité auquel les corps policiers doivent faire face. 
Ces constatations sont d'autant plus valables que, pour la période étudiée, les taux de solution sont restés presque partout quasi stables (avec une faible décroissance particulièrement évidente dans la Communauté urbaine de Montréal) alors que la criminalité n'a cessé de grimper.

De plus, entre 1962 et 1980 , l'augmentation annuelle moyenne du nombre des personnes accusées pour vol qualifié dans les cinq zones métropolitaines se chiffre à $15,6 \%$ dans la Communauté urbaine de Montréal, $8,7 \%$ pour la zone de Toronto et $23,6 \%$ pour la zone de Vancouver. Cette dernière surclasse de loin la Communauté urbaine de Montréal.

Lors de la seconde étape de cette recherche, soit l'évaluation de l'action policière, la variation des deux indicateurs de l'efficacité policière, à savoir taux de solution et taux de personnes accusées par rapport aux taux de criminalité (vol qualifié et vol à main armée), a révélé que l'augmentation du volume de criminalité est indépendante des efforts consacrés par la police dans le domaine du taux de solution et du nombre des personnes accusées.

La tentative effectuée par Wellford (1974) pour évaluer l'impact de l'effort de la police, mesuré par le taux de solution sur le taux de criminalité, a révélé une relation insignifiante entre les deux faits. Par ailleurs, les résultats obtenus par Chilton (1982) contredisent l'hypothèse des effets dissuasifs du taux d'arrestation sur le taux de criminalité. Nos résultats, malgré l'utilisation d'une méthode d'analyse peu complexe corroborent donc, jusqu'à un certain point, les conclusions de plusieurs qui se sont penchés avant nous sur ce problème épineux «police et criminalité».

Cependant, si nous acceptons qu'il n'y ait pas de relation entre le nombre de crimes commis (vol qualifié et vol à main armée) et le nombre de crimes solutionnés, ou encore le nombre de crimes commis et celui des personnes accusées pour ces crimes, ceci ne veut pas dire que la répression policière reste passive devant la montée de la criminalité.

En effet, au Québec et dans la Communauté urbaine de Montréal, le rapport entre le taux de personnes accusées et le taux de criminalité a montré une légère tendance à la fois à la baisse et à la stabilisation. Cela signifie que des changements parallèles se sont produits dans les taux de criminalité et dans les taux de personnes accusées, avec des taux de criminalité augmentant plus 
rapidement que ceux des taux des personnes accusées. De plus, en comparant les données sur les vols qualifiés avec celles du nombre des personnes accusées, nous avons constaté que les vols qualifiés commis au Québec sont trois fois supérieurs en nombre à celui de personnes accusées. Parallèlement, l'analyse a révélé que dans les zones métropolitaines où les vols qualifiés et les vols à main armée sont moins nombreux, les corps policiers enregistrent un taux relativement élevé de vols qualifiés ou de vols à main armée solutionnés. Tel est le cas de la province d'Ontario et de sa zone métropolitaine de Toronto ou encore de la province d'Alberta et de ses zones métropolitaines.

Une situation semblable s'est présentée au Québec et dans la Communauté urbaine de Montréal en 1966, où les données ont montré des taux de solution de vols qualifiés élevés $(37,4 \%)$, pour le Québec; $32,3 \%$ pour Montréal) alors que les taux de crimes enregistrés étaient les plus bas.

En ce qui a trait au taux de solution des vols à main armée, les territoires faisant l'objet de l'étude présentent les situations suivantes :

Ontario et Toronto métropolitain : Taux de crimes solutionnés élevés, criminalité en baisse.

Alberta et Calgary métropolitain : Taux de crimes solutionnés élevés, criminalité faible.

Colombie britannique et Vancouver métropolitain : Taux de crimes solutionnés relativement faibles (selon les années), criminalité relativement faible.

Québec et Communauté urbaine de Montréal : Taux de crimes solutionnés faibles, criminalitê excessivement élevée.

Ajoutons qu'au cours de l'année 1975, où les vols à main armée étaient en hausse dans tous les territoires concernés, le taux de solution déclaré par les corps policiers n'a pas changé de façon notoire. De plus, en 1978, toutes les provinces et zones métropolitaines ont enregistré le plus fort taux de solution alors que pour la même année, les vols à main armée étaient en baisse partout.

Ces constatations nous amènent à penser que plus le volume de criminalité augmente, plus l'écart, entre le taux de criminalité 
et le taux de solution ou taux des personnes accusées pour vols qualifiés et vols à main armée, s'élargit et ce, malgré une répression policière constante. Donc, une des conséquences logiques de l'augmentation du taux de criminalité pourrait s'exprimer par une diminution du taux de solution malgré les efforts fournis par la police.

Selon nos résultats, c'est surtout l'action de la police de la Communauté urbaine de Montréal, en matière de vol qualifié et de vol à main armée, qui répond à un tel schéma.

Ainsi, au lieu de conclure à l'inefficacité de son action, relativement au contrôle de ces crimes spécifiques, il nous semble plus raisonnable de penser que la police de la Communauté urbaine de Montréal s'est trouvée devant la montée rapide du volume de ce genre de criminalité.

Or, si l'on parle de la saturation du travail policier à cause d'une augmentation rapide du volume de criminalité, l'on admet aussi que c'est le volume de criminalité qui détermine, au moins en partie, l'efficacité du contrôle policier vis-à-vis la criminalité.

Il serait donc hasardeux de vouloir résoudre la question de l'impact de l'action policière puisque, tel que nous l'avons vu, son efficacité n'est pas une donnée absolue mais apparaît comme étant relative au volume de criminalité. Par ailleurs, il faudrait identifier au préalable un seuil d'efficacité de ce service en matière du contrôle de la criminalité.

Tittle et Rowe (1974) avaient délimité ce seuil à un taux de solution de $30 \%$. À ce point critique, on constate des changements notoires dans le volume de la criminalité. De son côté, Bailey (1976) effectuant la même analyse, a vu ses résultats corroborer ceux de Tittle et Rowe. Néanmoins, la méthodologie rudimentaire utilisée dans la présente étude, ne nous permet pas de définir un tel seuil de l'efficacité de l'action policière.

L'ensemble des résultats semble appuyer l'hypothèse de l'existence d'un seuil de saturation et l'idée que ce seuil serait bas. En bref, un plus grand nombre de cas sont résolus lorsque la demande de service est relativement faible. Lorsque cette demande augmente, on enregistre assez vite une diminution proportionnelle du nombre d'incidents réglés par mise en accusation. Il s'agit d'une 
relation inverse assez claire. Elle a été si souvent constatée qu'il devient raisonnable de l'admettre et de la considérer comme décrivant une situation stable entre l'évolution de la criminalité et l'efficacité de l'action policière. Les figures 1 et 2 illustrent assez bien les faits et résument l'essentiel de ce qui précède.

\section{CONCLUSION}

Cette analyse ne fait que signaler, dans le cas d'un crime particulier, une situation plus générale et stable qu'on ne serait porté à l'imaginer. En effet, lorsqu'on réunit les données relatives à l'ensemble des villes canadiennes, on observe que, depuis 1962, le nombre des policiers en uniforme a régulièrement augmenté; le nombre de crimes signalés également; par contre, la proportion d'incidents résolus par la police par voie de mise en accusation a régulièrement diminuée (Hasenpusch, B., 1982). Telle est la trame de base et ceci semble difficilement réfutable. Or, il n'est pas indifférent que cette évidence soit clairement et souvent, énoncée et décrite. En effet, elle interpelle, à travers un constat d'échec, la croyance en la protection policière et ses conséquences.

Au bout du compte et à tout prendre, que nous conduisent à penser les faits observés? En premier lieu, évidemment, on admet que l'action policière n'a pas d'impact sensible sur l'évolution du type de criminalité discuté ici. Mais on est également porté à croire qu'elle ne peut pas en avoir. Et, il est possible que ce soit également vrai pour tous les types de crime.

Par ailleurs, poser la question de l'efficacité de la police, fait inévitablement surgir les considérations au sujet de ses fonctions. Or, à ce chapitre, on constate que ces dernières se sont fort diversifiées. Ainsi, depuis longtemps, la proportion des ressources consacrées directement à la répression de la criminalité est assez modeste. Dans ce contexte, on peut se demander s'il est encore pertinent de discuter de l'efficacité et de son évaluation. Il semble que non. Ainsi, il devient évident qu'il faille reconsidérer l'ensemble des sujets et examiner des questions préalables plus éclairantes. Maintenir la police dans sa forme actuelle signifie que la société continue d'armer et d'entretenir à des coûts exorbitants en termes d'argent et de pourvoir un service dont les fonctions et le rôle sont devenus ambigus. 
FIGURE 1

Taux par 100000 habitanis de vols qualifies dans les zones métropolitaines de

Montréal, Toronto, Vancouver, Calgary et Edmonton (1962-1980).

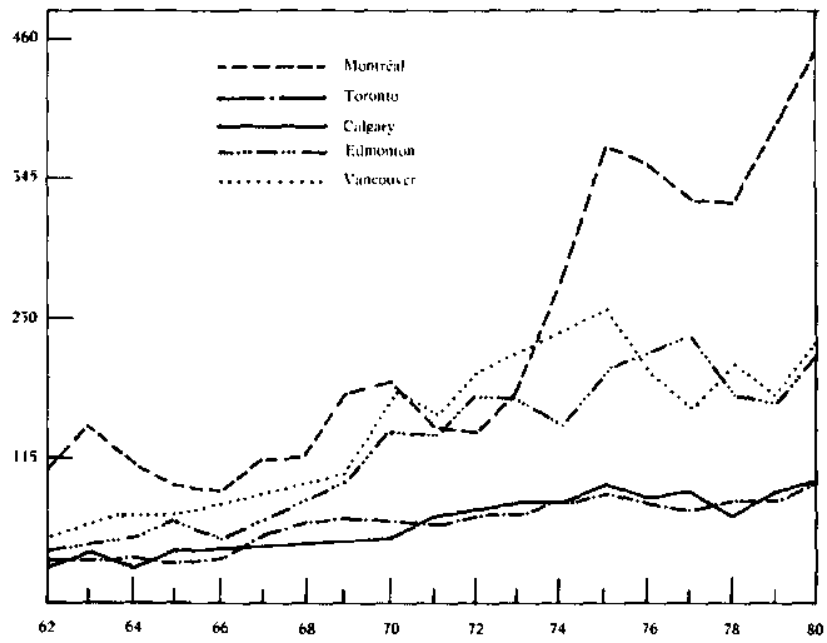

FIGURE 2

Estimation de taux de résolution des vols qualifiés dans les zones métropolitaines de

Montréal, Toronto, Vancouver, Calgary et Edmonton (1962-1980).

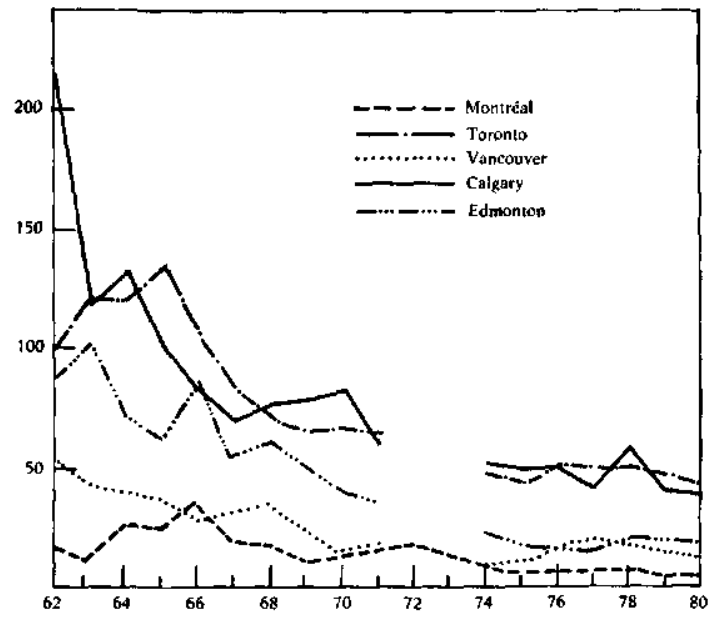




\section{BIBLIOGRAPHIE}

BAILEY, C.W. (1976), «Certainty of Arrest and Crime Rates for Major Felonies», A Research Note. Journal of Research in Crinte and Delinquency, 13, 2 (juillet), pp. 145-154.

BERNARD, A. et al. (1975), Profil, Toronto, Affaires urbaines, Canada. Ottawa.

BLOOM, J.N., P. CARROLL, (1967), «Police Effectiveness Measurement, Myth or Reality», Proceeding of the First Symposium, Law Enforcement Science and Technology, vol. 1, London Academy Press.

BLUMSTEIN, A.J. et al. (1978), Deterrence and Incapacitation Estimating the Effects of Criminal Sanctions on Crime rates, Washington D.C., National Academy of Sciences.

CHAMPMAN, I.J. (1976), «An Economic Model of Crime and Police : Some Empirical Results», Journal of Research in Crime and Delinquency, 13, 1, pp. 48-63.

CHAMPMAN, I.J. (1973), The Impact of Police on Crime and Crime on Police, A synthesis of the economic and ecological approaches, Institute of Government and Public Affaires UCLA.

CHILTON, R. (1982), "Analysing Urban Crime data», Criminology, 19, 4 (février), p. 590-607.

COMMISSION DE POLICE DU QUÉBEC, Rapport annuel 1980, Editeur officiel, 1981.

COMMUNAUTÉ URBAINE DE MONTRÉAL, Rapport annuel, 1972-1980.

CONKLIN, E.J. (1972), Robbery and the Criminal Justice System, Philadelphia, J.B. Lippincott.

ELIE, D. (1981), «La criminalité au Québec», Criminologie, 14, 1, pp. 85-104.

ELLIOTT, J.F. et T. SARDINO, (1971), Crime Control Team, An Experiment in Municipal Police Department Management and Operations, Springfield, Ill., Thomas.

FATTAH, E.A. (1973), Étude de criminologie écologique: La criminalité dans les zones métropolitaines au Canada, Bilan d'une décennie (1962-1971), Ecole de criminologie, Université de Montréal.

FEENEY, F. et A. WERR, (1975), \&Prevention and Control of Robbery», Criminology, 13, 1, mai, pp. 102-105.

GERMAN, A. et al. (1962), Introduction to Law Enforcemnet, Springfield, III., Thomas.

GREENBERG et al. (1973), «A Panel Model of Crime Rates and Arrest Rates», American Sociological Review, 44, octobre, pp. 843-850.

GRIFFITH et al. (1980), Criminal Justice in Canada. An Introductory Text, Scarborough, Ont., Butterworths.

GROUPE DE TRAVAIL SUR LE VOL A MAIN ARMÉE (1980), le Vol à main armée au Québec, Québec, ministère de la Justice. 
GROUPE DE TRAVAIL SUR I'ORGANISATION ET LES FONCTIONS POLICIÉRES (1978), (Rapport Saulnier), Éditeur officiel du Québec.

HASENPUSCH, B. (1982), Future Trends in Crime and Crime Control in Canada, Thèse de doctorat non publiée, Université de Montréal, Ecole de criminologie.

HATRY, P.H. (1975), «Wrestling with Police Control Productivity Measurement», Readings on Productivity in Policing, edited by Joan L. Wolfle and J.F. Heaphy, pp. 86-128.

LOFTIN, C. et D. McDOWALL, (1982), «The Police, Crime and Economic Theory: An Assessment», American Sociological Review, 47, juin, pp. 393-401.

MARX, H. (1981), la Police au Québec: Son contrôle et son coat, École de criminologie, Université de Montréal, $30 \mathrm{p}$.

Mémoire présenté à la partie patronale par la Fraternité des policiers de la C.U.M. les 16 et 17 janvier 1982, Cheribourg, Magog.

Metropolitain Toronto Department, Metropolitain Toronto Department Statistical Report, 1972 à 1977 et 1979 à 1980, annual.

NAGIN, D. (1978), «General Deterrence : A Review of the Empirical Evidence», dans Alfred Blumstein, Jacqueline Cohen and Daniel Nagin (édit), Deterrence and Incapacitation : Estimating the Effect of Criminal Sanctions on Crime Rates, Washington, D.C., National Academy of Science.

NATIONAL ADVISORY COMMISSION ON CRIMINAL JUSTICE STANDARDS AND GOALS (1973), Police Task Force on Police, Washington, D.C., 9-57, pp. 101-151.

NEEDLE, J.A. et M.W. O'NEIL, «L'évaluation de la performance des programmes de police, Schéma pour jauger l'efficacité et la productivité», dans Compte rendu l'Atelier sur la productivité de la police, Solliciteur général du Canada, Division de la recherche, pp. 40-91.

NORMANDEAU, A. et D.D. AKMAN, (1966), A Manual for Constructing a Crime and Delinquency Index in Canada, Montréal, University of Montréal, Department of Criminology.

PHILLIPS, L. et H. VOTEY, jr (1972), «An Economic Analysis of the Deterrent Effect of Law Enforcement on Criminal Activities», Journal of Criminal Law, Criminology and Police Science, 63 (3), pp. 336-342.

PONTELL, H.N. (1978), «Deterrence : «Theory versus Practice», Criminology, 16, pp. 3-22.

REISS, J.A. jr. (1971), The Police and the Public, New Haven, Yale University Press.

RAPPORT OUIMET (1969), Justice pénale et correction : un lien à forger, Ottawa, Imprimeur de la reine.

SKOGAN, N. (1976), «Efficiency and Effectiveness in Big City Police Departments», Public Administration Review, 3 mai-juin, pp. 278-286.

STATISTIQUE CANADA, Statistiques de la criminalité 1962-1980, Catalogue no 85-205, annuel, Ottawa.

STATISTIQUE CANADA, Statistigues de l'administration policière 1962. 1972, Catalogue n० 85-204. 
STATISTIQUE CANADA, Révision des estimations annuelles de la population selon le sexe et l'âge 1921-1971, Catalogue no 91-512, hors série, Ottawa.

STATISTIQUE CANADA, Population, révision des estimations annuelles de la population selon le sexe et l'âge 1971-1976. Catalogue no 91-518, hors série, Ottawa.

STATISTIQUE CANADA, Estimation de la population selon le sexe et l'âge 1977-1980, Catalogue no 91-202, annuel depuis 1977, Ottawa.

SUTHERLAND, E.H. et D.R. CRESSEY (1966), aLes indices de la criminalité dans leur livre», Principes de criminologie, Paris, Cujas, pp. 33-59.

SWIMMER (1974), «The Relationship of Police and Crime, some Methodological and Empirical Results», Criminology, 12, 3, pp. 293-314.

TASK FORCE REPORT (1967), Crime and its Impact - An Assessment, The president's Commission on Law Enforcement and Administration of Justice, Washington, D.C., 220 p.

TERRENCE, T. (1974), «The Impact of Crime Rate, Changes on Police Protection Expenditures in American Cities», Criminology, 11, 4, pp. 512-525.

TITTLE, C.R. (1969), «Crime Rates and Legal Sanctions», Social Problems, 16 , printemps, pp. $409-423$.

TITTLE, C.R. et A.R. ROWE, (1974), «Certainty of Arrest and Crime Rates : A Further Test of the Deterrence Hypothesis», Social Forces, 52, juin, pp. 455-462.

VOTEY, H., Ph. LIAD, (1974), «The Control of Criminal Activity an Economic Analysis», Daniel Glaser (édit.), Handbook of Criminology, Chicago, Rand McNally.

WELlFORD, C.R. (1974), «Crime and the Police», Criminology, 12, pp. 195-213.

WESTLEY, W.A. (1970), «Violence and the Police», A Sociological Study, Cambridge, M.I.T. Press.

WILSON, J.Q., B. BOLAND, (1979), The Effect of the Police on Crime, Washington, D.C., Department of Justice. 